MOMO, C. e WERTHER, K. Parasitismo renal em perdizes (Rhynchotus rufescens) criadas em cativeiro. PUBVET, Londrina, V. 7, N. 16, Ed. 239, Art. 1578, Agosto, 2013.

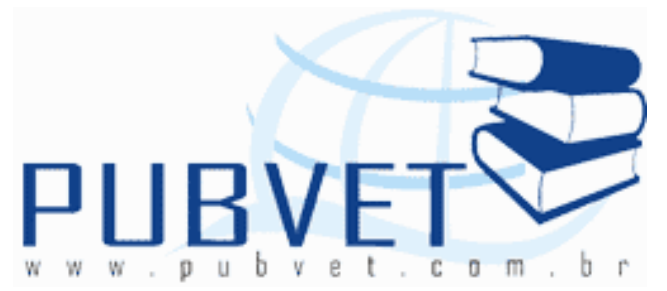

PUBVET, Publicações em Medicina Veterinária e Zootecnia.

\title{
Parasitismo renal em perdizes (Rhynchotus rufescens) criadas em cativeiro
}

Claudia Momo ${ }^{1}$ e Karin Werther ${ }^{2}$

\begin{abstract}
${ }^{1}$ Aluna de doutorado na área de Patologia Animal na UNESP - Campus de Jaboticabal.

2 Professora do Departamento de Patologia Veterinária na UNESP - Campus de Jaboticabal. Departamento de Patologia Veterinária. Faculdade de Ciências Agrárias e Veterinárias. Via de Acesso Prof. Paulo Donato Castellane, s/n Zona Rural. CEP 14884-900. Jaboticabal/SP, Brasil. E-mail: werther@fcav.unesp.br
\end{abstract}

\section{Resumo}

A perdiz (Rhynchotus rufescens), pertencente à família Tinamidae, é uma ave que apresenta relativa facilidade à adaptação ao cativeiro. É considerada apta à produção de carne de boa qualidade e possui uma ótima conversão alimentar. Essas características a tornam interessante para produção comercial. Visando conhecer as principais afecções que acometem essas aves em cativeiro, foram analisados achados macro e microscópicos de 62 perdizes que vieram a óbito ao longo de 12 anos, provenientes de criatório experimental, mantidas em recintos de terra. Os exames anatomopatológicos revelaram parasitismo renal pelo trematoda Paratanaisia confusa em $16,1 \%$ dos animais. Entre as perdizes parasitadas, 30\% também apresentaram gota úrica, como provável consequência 
MOMO, C. e WERTHER, K. Parasitismo renal em perdizes (Rhynchotus rufescens) criadas em cativeiro. PUBVET, Londrina, V. 7, N. 16, Ed. 239, Art. 1578, Agosto, 2013.

da obstrução dos túbulos coletores pelo parasito. O objetivo deste trabalho foi descrever a infestação pela Paratanaisia confusa em perdizes criadas em cativeiro.

\title{
Renal parasitism in red winged tinamou (Rhynchotus rufescens) raised in captivity
}

\begin{abstract}
The partridge (Rhynchotus rufescens), belonging to the family Tinamidae, is a bird that has relatively easy adaptation to captivity. It is considered suitable for meat production of good quality and has a great feed. These characteristics make it interesting for commercial production. Aiming to know the major diseases that affect these birds in captivity, were analyzed gross and microscopic findings of 62 partridges that died over 12 years, from experimental breeding, kept in enclosures of land. The pathology revealed renal parasitism by Trematoda Paratanaisia confusa in $16.1 \%$ of the animals. Among partridges parasitized, $30 \%$ also had gout uric as probable consequence of obstruction of the collecting tubules by the parasite. The aim of this study was to describe the infestation by Paratanaisia confusa in captive bred partridges.
\end{abstract}

\section{INTRODUÇÃO}

De acordo com dados oficiais brasileiros, nos últimos anos o número de mantenedouros conservacionistas e criatórios comerciais de perdizes no Brasil tem aumentado, compreendendo atualmente 13 criatórios (IBAMA, 2012). Considerando a aptidão zootécnica dessas aves (MORO, 2001), é importante conhecer as doenças que prejudicam o seu desenvolvimento. São poucos os artigos publicados referentes às doenças que acometem esses animais, entre eles, há um estudo retrospectivo de exames post mortem em perdizes, no qual a gota úrica foi a alteração mais frequente (WERTHER, 2003). 
MOMO, C. e WERTHER, K. Parasitismo renal em perdizes (Rhynchotus rufescens) criadas em cativeiro. PUBVET, Londrina, V. 7, N. 16, Ed. 239, Art. 1578, Agosto, 2013.

Com o crescente interesse na criação e a falta de literatura específica, o objetivo deste trabalho foi descrever, em perdizes criadas em cativeiro, a presença do trematoda Paratanaisia confusa.

\section{MATERIAL E MÉTODOS}

As 62 aves utilizadas nessa pesquisa foram provenientes de um criatório experimental da Faculdade de Ciências Agrárias e Veterinárias (FCAV) da Unesp Câmpus de Jaboticabal/SP. As aves eram mantidas em baias com telas de alambrado, piso de terra e touceiras de capim. A cobertura parcial era com telhas de cerâmica, para proteção contra chuva e sol. Em cada baia viviam 10 a 12 aves, entre machos e fêmeas. A alimentação oferecida era ração peletizada, contendo $24 \%$ de proteína bruta, produzida na própria Faculdade.

Os animais que vieram a óbito foram necropsiados no Departamento de Patologia Veterinária da FCAV. No exame anatomopatológico, inicialmente foi avaliado o escore corporal (musculatura peitoral e anel de gordura epicárdico), sendo classificado em adequado, magro ou caquético (MAXIE, 1993). Após avaliação macroscópica dos órgãos, foram colhidas amostras, fixadas em solução de formolina a 10\%, tamponada e processadas pelas técnicas de rotina histológica e coradas pela técnica de Hematoxilina-Eosina.

\section{RESULTADOS E DISCUSSÃO}

Em relação à avaliação do escore corporal, 68,8\% das aves estavam com massa muscular adequada e $21,9 \%$ magras e 9,3\% caquéticas.

Como achado macroscópico, a nefromegalia foi observada em $20 \%$ dos animais acometidos, concordando com os relatos de Luppi et al (2007) e Brener et al (2006). Já, contrariamente, Silva et al (2004), Gomes et al (2005) e Menezes et al (2001), referem a ausência de lesões macroscópicas renais.

Entre os achados microscópicos encontrados nos rins, 16,1\% (10/62) das perdizes apresentaram o parasito Paratainasia confusa nos ductos coletores 
MOMO, C. e WERTHER, K. Parasitismo renal em perdizes (Rhynchotus rufescens) criadas em cativeiro. PUBVET, Londrina, V. 7, N. 16, Ed. 239, Art. 1578, Agosto, 2013.

renais (Figura 1 A e B). O parasito foi identificado pelo Departamento de Medicina Veterinária Preventiva da FCAV/UNESP/Jaboticabal.

No Brasil são descritas três espécies de trematodas renais do gênero Paratainasia, ou seja: P. bragai, P. confusa e a P. robusta. Em perdizes, há relatos da ocorrência da $P$. confusa em cativeiro (MAPELI et al, 2003), e $P$. robusta em aves de vida livre (NASCIMENTO \& ARANTES, 2001). Gomes et al (2005) referem que a infestação pela $P$. bragai é mais frequentemente observada em animais das Ordens Columbiformes, Galliformes e Anseriformes. Rotstein et al (2005) incluem também os Passeriformes e Piciformes. Brener et al (2005) descreveram uma prevalência de $20 \%$ de $P$. bragai em perus e Silva et al (2004), de $31,8 \%$ em galinhas d'angola.

Rotstein et al (2005) referem que o ciclo de vida deste trematoda renal inclui uma fase de maturação dos adultos nos rins e a eliminação pela urina de ovos embrionados, que por sua vez são ingeridos por lesmas e caracóis. Segundo Arzinaut et al (1992), Brandolini et al (1997) e Gomes et al (2005), a infestação pela Paratanaisia sp. ocorre pela ingestão de caracóis e lesmas que abrigam as metacercárias. Estas são liberadas na luz intestinal das aves. Quando chegam na cloaca seguem pelos ureteres até os rins. As perdizes desse estudo estavam alojadas em piso de terra com grama, local propício para a presença dos hospedeiros intermediários e recontaminação ambiental.

Achados microscópicos concomitantes ao parasitismo renal foram a dilatação de ductos coletores em $100 \%$ das aves, alteração também descrita na maioria dos relatos (MENEZES et al, 2001; MAPELI et al, 2003; GOMES et al, 2005; BRENER et al., 2006; LUPPI et al., 2007). No entanto, Silva et al (2004) não constataram dilatação dos ductos coletores.

Foi observado moderado infiltrado inflamatório composto por linfócitos, macrófagos e heterófilos ao redor dos ductos coletores e no interstício de $80 \%$ dos animais. Menezes et al. (2001), Brener et al (2006) e Rotstein et al (2005) referem que a intensidade e o tipo de reação inflamatória compreendem desde 
MOMO, C. e WERTHER, K. Parasitismo renal em perdizes (Rhynchotus rufescens) criadas em cativeiro. PUBVET, Londrina, V. 7, N. 16, Ed. 239, Art. 1578, Agosto, 2013.

nefrite intersticial composta por macrófagos e heterófilos; infiltrado eosinofílico e linfocítico-plasmocitário (MAPELI et al, 2003), infiltrado linfocitário (GOMES et al, 2005), até nefrite granulomatosa (LUPPI et al., 2007, BUNBURY et al, 2008). De acordo com Brener et al (2006), a P. bragai causa espessamento da parede dos ductos coletores, fato também observado em $30 \%$ dos animais acometidos.
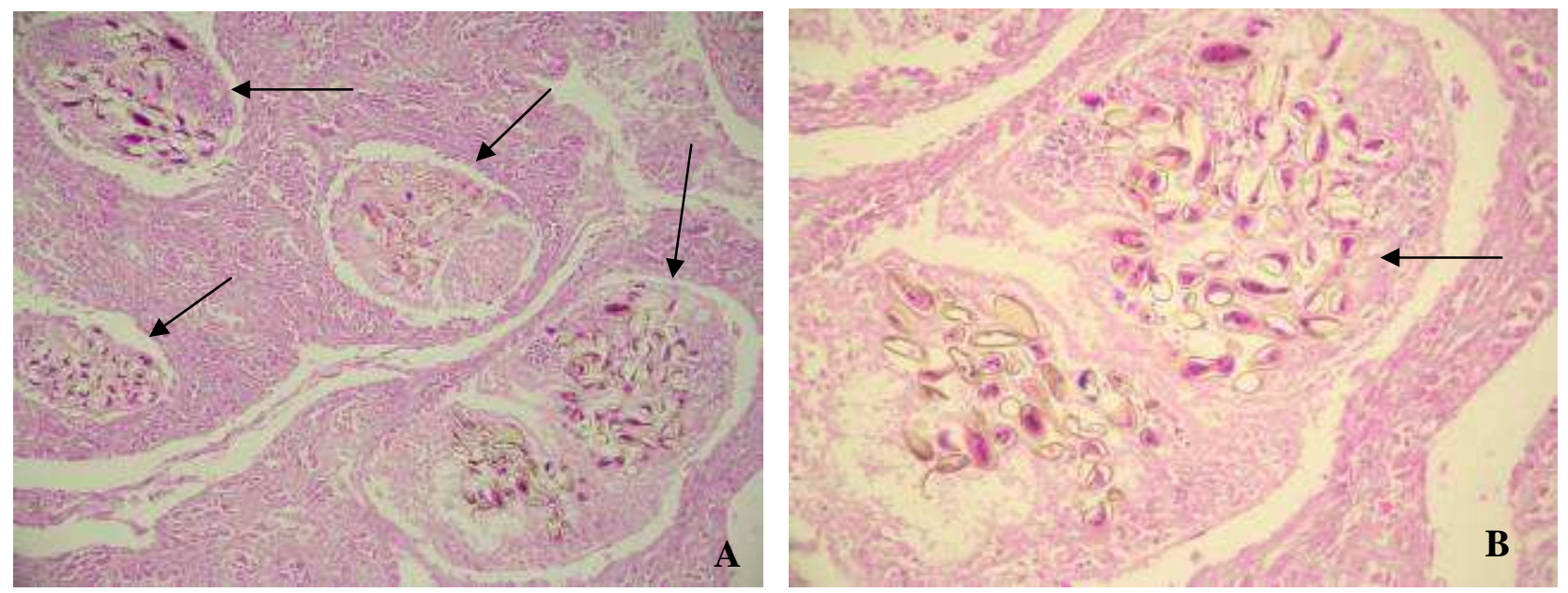

Figura 1: A e B: Fotomicrografia de corte histológico do rim de perdiz mostrando o parasito Paratanaisia confusa nos ductos coletores (seta). A: HE, 100 x. B: HE, $400 \mathrm{x}$.

Pinto et al (2004) reportam em uma espécie de columbiforme, destruição e achatamento das células epiteliais tubulares, o que não foi observado neste estudo. Já Luppi et al (2007) relataram a infestação de três espécies de psitacídeos, dois dos quais apresentaram infestação tanto pela $P$. bragai quanto pela $P$. robusta. Foi descrito, como achado secundário, a fibrose na região da pelve e ao redor dos ductos coletores. Achado que não foi verificado neste estudo.

No atual trabalho, os parasitos foram observados na luz dos ductos coletores, podendo causar obstrução, de acordo com GOMES et al (2005). Por outro lado, (PINTO et al, 2004) referem que a presença do parasito leva a uma dilatação compensatória dos ductos coletores, não induzindo a retenção de urina. 
MOMO, C. e WERTHER, K. Parasitismo renal em perdizes (Rhynchotus rufescens) criadas em cativeiro. PUBVET, Londrina, V. 7, N. 16, Ed. 239, Art. 1578, Agosto, 2013.

Luppi et al (2007) encontraram o parasito também no interstício. Brener et al (2006) observaram dilatação dos ductos coletores renais com espessamento da parede e infiltrado inflamatório. Essa variação de lesões reforça a variação de patogenicidade nas diferentes espécies de aves (Gomes et al, 2005).

Apesar de o parasito ser considerado pouco patogênico por Pinto et al (2004) e Gomes et al (2005), no presente estudo das 10 aves que apresentaram infestação por $P$. confusa, 30\% também apresentaram gota úrica, sugerindo uma possível relação direta entre parasitismo e indução de lesões secundárias renais. Em aves, a gota é observada em decorrência de afecções renais prévias (TUNG et al, 2006). O efeito paralelo da presença do parasita nos ductos coletores leva a obstrução parcial dos mesmos, ocasionando a não excreção e o acúmulo de cristais de ácido úrico nos túbulos renais, originando a gota úrica. A gota úrica visceral é descrita como um resultado de um severo e rápido desenvolvimento de hiperuricemia secundário a uma nefropatia obstrutiva (obstrução renal), com a precipitação de cristais de ácido úrico nos túbulos renais, ductos coletores ou ureteres (LUMEIJ, 1994). Quando a função renal está abaixo de 30\% da capacidade original, há um aumento na concentração de ácido úrico sérico e deposição nos tecidos (TUNG et al, 2006; MEYERHOLZ et al., 2005).

Moro et al (2002) referem 15\% como uma quantidade ideal de proteína bruta na ração de manutenção para perdizes), A alta taxa de proteína na ração (24\%) utilizada nesse trabalho, assim como o parasitismo por P.confusa, podem ser considerados fatores predisponentes para a ocorrência da gota úrica. O excesso de proteína pode desencadear um quadro de hiperuricemia, com deposição sistêmica de cristais de ácido úrico (LUMEIJ, 1993). Isto sugere uma relação entre o desenvolvimento de gota úrica e a quantidade de proteína bruta na dieta, fato também relatado por Goodman (1996) e Lumeij (1994). 
MOMO, C. e WERTHER, K. Parasitismo renal em perdizes (Rhynchotus rufescens) criadas em cativeiro. PUBVET, Londrina, V. 7, N. 16, Ed. 239, Art. 1578, Agosto, 2013.

\section{CONCLUSÕES}

Considerando o ciclo do trematoda em questão, com a presença de hospedeiros intermediários e o tipo de recinto, conclui-se que o recinto com piso de terra favorece o ciclo desse parasito. Quanto à gota úrica, esta pode ser resultado da associação de dois fatores, a obstrução parcial dos túbulos coletores pelo parasito e a possível hiperuricemia, provocada pela alta taxa de proteína bruta na ração. Para prevenir o parasitismo renal na criação de perdizes (Rhynchotus rufescences), as aves não devem ter acesso a terra, nem aos hospedeiros intermediários. Para reduzir os índices de gota úrica é importante adequar os níveis de proteína na dieta.

\section{BIBLIOGRAFIA}

Instituto Nacional do Meio Ambiente e dos Recursos Naturais Renováveis (IBAMA). <www.ibama.gov.br/fauna/criadouros/comerciais.pdf> Acesso em 20 de novembro de 2012.

BRANDOLINI, S.V.P.B.; AMATO, S.; PEREIRA, A.A. Relacionamento de Tanaisia bragai (Digenea, Eucotylidae) e seu hospedeiro intermediário, Subulina octona (Gastropoda, Subulinidae) sob condições experimentais. Parasitologia al Dia, Santiago,1997, 21, 109-13.

BRENER, B.; TORTELLY, R.; MENEZES, R.C.; MUNIZ-PEREIRA, L.C.; PINTO, R.M. Prevalence and pathology of the nematode Heterakis gallinarum, the trematode Paratanaisia bragai, and the protozoan Histomonas meleagridis in the turkey, Meleagris gallopavo. Mem Inst Oswaldo Cruz, Rio de Janeiro, Vol. 101(6): 677-81, September 2006.

BUNBURY, N.; STIDWORTHY, M.F.; GREENWOOD, A.G.; JONES, C.G.; SAWMY, S.; COLE, R.E.; EDMUNDS, K.; BELL, D.J. Causes of mortality in free-living Mauritian pink pigeons Columba mayeri, 2002-2006. Endangered species research. 2008. On line. 23 de abril. doi: 10.3354/esr00088.

DEHGHAN, A.; KÖTTGEN, A.; YANG, Q.; HWANG, S.J.; LINDA KAO, W.H.; RIVADENEIRA, F.; BOERWINKLE, E.; LEVY, D.; HOFMAN, A.; ASTOR, B.C.; BENJAMIN, E.J.; DUIJN, C.M.; WITTEMAN, J.C.; CORESH, J.; FOX, C.S. Association of three genetic loci with uric acid concentration and risk of gout: a genome-wide association study. Lancet, 2008, 372, 1953-61.

GOMES, D.C; MENEZES, R.C.; TORTELLY, R.; PINTO, R.M. Pathology and first occurrence of the kidney trematode Paratanaisia bragai (Santos, 1934) Freitas, 1959 (Digenea, Eucotiylidae) in Phasianus colchicus L., 1758 from Brazil. Mem. Inst. Oswaldo Cruz, 2005, 100(3), 285-88.

GOODMAN, G.J. Metabolic disorders. In: ROSSKOPF, W.; WOERPEL, R., eds. Diseases of cage and aviary birds. 3rd ed. Philadelphia, PA: Williams \& Wilkins, 1996, 477-78.

LUMEIJ, J.T. Nephrology. In: RITCHIE, B.W.; HARRISSON, G.J.; HARRISSON, L.R., eds. Avian medicine: principles and application. Lake Worth, FL: Wingers, 1994, 538-55. 
LUPPI, M.M.; DE MELO, A.L.; MOTTA, R.O.C.; MALTA, M.C.C.; GARDINER, C.H.; SANTOS, R.L. Granulomatous nephritis in psittacines associated with parasitism by the trematode Paratanaisia $s p$. Veterinary Parasitology, v.146, p. 363-366, 2007.

MAPELI, E.B., NASCIMENTO, A.A., SZABÓ, M.P.J., TEBALDI, J.H. Infecções naturais por helmintos em perdizes (Rhynchotus rufescens Temminck, 1815) de cativeiro, no município de Jaboticabal, Estado de São Paulo. Arq. Inst. Biol., São Paulo, 2003, 70(4), 415-418.

MAXIE, G.M. The urinary sistem. In: JUBB, K.V.F.; KENNEDY, P.C.; PALMER, N. Pathology of domestic animals. $4^{\text {th }}$ ed. San Diego, CA: Academic Press, 1993, 447-538.

MEYERHOLZ, D.K.; VANLOUBBEECK, Y.F.; HOSTETTER, S.J.; JORDAM, D.M.; FALES-WILLIAMS, A.J. Surveillance of amyloidosis and other diseases at necropsy in captive trumpete swans (Cygnus buccinator). J Vet Diag Invest, 2005, 17, 295-298.

MORO, M.E.G.; ARIKI, J.; MALHEIROS, E.B. Avaliação dos níveis de proteína da dieta sobre a idade à maturidade sexual e produção de ovos de perdiz (Rhynchotus rufescens Temminek). Acta Scientiarum. Maringá, v. 24, n. 4, p. 997-1000, 2002.

MORO, M.E.G. Red winged tinamou (Rhynchotus rufescens) - utilization for meat production. In: FOWLER, M.E.; CUBAS, Z.S. Biology, Medicine and Surgery of South American Wild Animals. Iowa State University, 2001: 75-76.

NASCIMENTO, A.A.; ARANTES, I.G. Helminthiasis of Tinamous. In: FOWLER, M.E.; CUBAS, Z.S. Biology, Medicine, and Surgery of South American Wild Animals. Iowa State University Press, 2001, 76-80.

PINTO, R.M.; MENEZES, R.C; TOTELLY, R. Systematic and pathologic study of Paratanaisia bragai (Santos, 1934) Freitas, 1959 (Digenea, Eucotylidae) infestation on ruddy ground dove Columbina talpacoti (Temminck, 1811). Arq. Bras. Med. Vet. Zootec., 2004, 56(4), 472-79.

TUNG, J.; MULLIN, M.; HEATLEY, J.J. What is your diagnosis? Journal of Avian Medicine and Surgery, v.20, n.1, p.39-43, 2006.

WERTHER, K. Principais enfermidades de perdizes. SIMPERDIZ, Jaboticabal, 2003. CD ROM. 